\title{
A GENERALIZED SAMPLING THEOREM OVER GALOIS FIELD DOMAINS FOR EXPERIMENTAL DESIGN
}

\author{
Yoshifumi Ukita \\ Department of Management and Information, \\ Yokohama College of Commerce, Yokohama, Japan \\ ukita@shodai.ac.jp
}

\begin{abstract}
In this paper, the sampling theorem for bandlimited functions over $G F(q)^{n}$ domains is generalized to one over $\prod_{i=1}^{n} G F\left(q_{i}\right)$ domains. The generalized theorem is applicable to the experimental design model in which each factor has a different number of levels and enables us to estimate the parameters in the model by using Fourier transforms. Moreover, the relationship between the proposed sampling theorem and orthogonal arrays is also provided.
\end{abstract}

\section{KEYWORDS}

Digital Signal Processing, Sampling Theorem, Experimental Design, Orthogonal Arrays, Fourier Analysis

\section{INTRODUCTION}

In digital signal processing [3], the sampling theorem states that any real valued function $f$ can be reconstructed from a sequence of values of $f$ that are discretely sampled with a frequency at least twice as high as the maximum frequency of the spectrum of $f$. This theorem can also be applied to functions over finite domain [4] [8]. For example, Ukita et al. obtained a sampling theorem over $G F(q)^{n}$ domains [8], which is applicable to the experimental design model in which all factors have the same number of levels. However, this sampling theorem is not applicable to the model in which each factor has a different number of levels, even though they often do [2], [7]. Moreover, a sampling theorem for such a model has not been provided so far. In this paper, the sampling theorem for bandlimited functions over $G F(q)^{n}$ domains is generalized to one over $\prod_{i=1}^{n} G F\left(q_{i}\right)$ domains. The generalized theorem is applicable to the experimental design model in which each factor has a different number of levels and enables us to estimate the parameters in the model using Fourier transforms. In addition, recently, the volume of the data has grown up rapidly in the field of Big Data and Cloud Computing [11] [12], and the generalized theorem can also be used to estimate the parameters for Big Data efficiently. Moreover, the relationship between the proposed sampling theorem and orthogonal arrays [1] is provided.

David C. Wyld et al. (Eds) : NETCOM, NCS, WiMoNe, CSEIT, SPM - 2015

pp. 237-247, 2015. (C CS \& IT-CSCP 2015

DOI : $10.5121 / \mathrm{csit} .2015 .51620$ 


\section{PRELIMINARIES}

\subsection{Fourier Analysis on Finite Abelian Groups}

Here, a brief explanation of Fourier analysis on finite Abelian groups is provided. Characters are important in the context of finite Fourier series.

\subsubsection{Characters [5]}

Let $G$ be a finite Abelian group (with the additive notation), and let $S^{1}$ be the unit circle in the complex plane. A character on $G$ is a complex-valued function $\chi: G \rightarrow S^{1}$ that satisfies the condition

$$
\chi\left(x+x^{\prime}\right)=\chi(x) \chi\left(x^{\prime}\right) \quad \forall x, x^{\prime} \in G .
$$

In other words, a character is a homomorphism from $G$ to the circle group.

\subsubsection{Fourier Transform [4]}

Let $G_{i}, i=1,2, \cdots, n$, be Abelian groups of respective orders $\left|G_{i}\right|=g_{i}, i=1,2, \cdots n, g_{1} \leq g_{2} \leq$ $\cdots \leq g_{n}$, and

$$
G=\times_{i=1}^{n} G_{i} \text { and } g=\prod_{i=1}^{n} g_{i} .
$$

Since the character group of $G$ is isomorphic to $G$, we can index the characters by the elements of $G$, that is, $\left\{\chi_{\boldsymbol{w}}(\boldsymbol{x}) \mid \boldsymbol{w} \in G\right\}$ are the characters of $G$. Note that $\chi_{\mathbf{0}}(\boldsymbol{x})$ is the principal character, and it is identically equal to 1 . The characters $\left\{\chi_{\boldsymbol{w}}(\boldsymbol{x}) \mid \boldsymbol{w} \in G\right\}$ form an orthonormal system:

$$
\frac{1}{g} \sum_{x \in G} \chi_{w}(x) \chi_{z}^{*}(x)= \begin{cases}1, & w=z \\ 0, & w \neq z\end{cases}
$$

where $\chi_{\boldsymbol{z}}^{*}(\boldsymbol{x})$ is the complex conjugate of $\chi_{\boldsymbol{z}}(\boldsymbol{x})$.

Any function $f: G \rightarrow \mathbb{C}$, where $\mathbb{C}$ is the field of complex numbers, can be uniquely expressed as a linear combination of the following characters:

where the complex number

$$
f(x)=\sum_{w \in G} f_{w} \chi_{w}(x)
$$

is the $\boldsymbol{w}$-th Fourier coefficient of $f$.

$$
f_{w}=\frac{1}{g} \sum_{x \in G} f(x) \chi_{w}^{*}(x),
$$




\subsection{Fourier Analysis on $\prod_{i=1}^{n} G F\left(q_{i}\right)$}

Assume that $q_{i}, i=1,2, \cdots n$, are prime powers. Let $G F\left(q_{i}\right), i=1,2, \cdots n$, be a Galois fields of respective orders $q_{i}, i=1,2, \cdots n$, which contain finite numbers of elements. We also use $\prod_{i=1}^{n} G F\left(q_{i}\right)$ to denote the set of all $n$-tuples with entries from $G F\left(q_{i}\right), i=1,2, \cdots n$. The elements of $\prod_{i=1}^{n} G F\left(q_{i}\right)$ are expressed as vectors.

Example 1: Consider $G F(2)=\{0,1\}$ and $G F(3)=\{0,1,2\}$. Then, if $n=3$ and $q_{1}=2, q_{2}=$ $2, q_{3}=3$,

$$
\prod_{i=1}^{3} G F\left(q_{i}\right)=\{000,001,002,010,011,012,100,101,102,110,111,112\}
$$

Specifying the group $G$ in Sect. 2.1 .2 to be the group of $\prod_{i=1}^{n} G F\left(q_{i}\right)$ and $g=\prod_{i=1}^{n} q_{i}$, the relations (3),(4) and (5) also hold over the $\prod_{i=1}^{n} G F\left(q_{i}\right)$ domain.

Then, the characters $\left\{\chi_{\boldsymbol{w}}(\boldsymbol{x}) \mid \boldsymbol{w} \in \prod_{i=1}^{n} G F\left(q_{i}\right)\right\}$ form an orthonormal system:

$$
\frac{1}{\prod_{i=1}^{n} q_{i}} \sum_{x \in \prod_{i=1}^{n} G F\left(q_{i}\right)} \chi_{\boldsymbol{w}}(\boldsymbol{x}) \chi_{\mathbf{z}}^{*}(\boldsymbol{x})= \begin{cases}1, & \boldsymbol{w}=\mathbf{z}, \\ 0, & \boldsymbol{w} \neq \mathbf{z},\end{cases}
$$

Any function $f: \prod_{i=1}^{n} G F\left(q_{i}\right) \rightarrow \mathbb{C}$, can be uniquely expressed as a linear combination of the following characters:

where the complex number

$$
f(\boldsymbol{x})=\sum_{\boldsymbol{w} \in \prod_{i=1}^{n} G F\left(q_{i}\right)} f_{w} \chi_{w}(\boldsymbol{x})
$$

$$
f_{\boldsymbol{w}}=\frac{1}{\prod_{i=1}^{n} q_{i}} \sum_{\boldsymbol{x} \in \prod_{i=1}^{n} G F\left(q_{i}\right)} f(\boldsymbol{x}) \chi_{\boldsymbol{w}}^{*}(\boldsymbol{x})
$$

is the $\boldsymbol{w}$-th Fourier coefficient of $f$.

\section{EXPERIMENTAL DESIGN}

In this section, a short introduction to experimental design [2], [7] is provided.

\subsection{Experimental Design Model}

Let $F_{1}, F_{2}, \cdots, F_{n}$ denote the $n$ factors to be included in an experiment. The levels of factor $F_{i}$ can be represented by $G F\left(q_{i}\right)$, and the level combinations can be represented by the $n$-tuples $\boldsymbol{x}=\left(x_{1}, x_{2}, \cdots, x_{n}\right) \in \prod_{i=1}^{n} G F\left(q_{i}\right)$.

\section{Example 2:}

Let Machine $\left(F_{1}\right)$ and Worker $\left(F_{2}\right)$ be factors that might influence the quantity of a product. $F_{1}$ : new machine (level 0), old machine (level 1), 
$F_{2}$ : highly skilled worker (level 0), average skilled worker (level 1), unskilled worker (level 2).

For example, $\boldsymbol{x}=01$ represents a combination of new machine and average skilled worker.

Then, the effect of the machine, averaged over all workers, is referred to as the effect of main factor $F_{1}$. Similarly, the effect of the worker, averaged over both machines, is referred to as the effect of main factor $F_{2}$. The contrast between the effect of the machine for a highly skilled worker, the effect of the machine for an average skilled worker, and the effect of the machine for an unskilled worker is referred to as the effect of the interaction of $F_{1}$ and $F_{2}$.

Next, an explanation of the model in the context of experimental design is given. In previous works [8], [9], [10], all factors were restricted to have the same number of levels. In this paper, I give the definition of the generalized model in which each factor has a different number of levels as follows.

\section{Definition 1: Generalized Model}

$\mathrm{y}(\mathrm{x})$ is used to denote the response of the experiment with level combination $\mathrm{x}$ and assume the model

where

$$
y(\boldsymbol{x})=\sum_{\boldsymbol{w} \in I_{A}} f_{w} \chi_{w}(\boldsymbol{x})+\epsilon_{\boldsymbol{x}},
$$

$$
I_{A}=\left\{\left(\mathrm{b}_{1} \mathrm{a}_{1}, \mathrm{~b}_{2} \mathrm{a}_{2}, \ldots, \mathrm{b}_{\mathrm{n}} \mathrm{a}_{\mathrm{n}}\right) \mid \boldsymbol{a} \in A, \mathrm{~b}_{\mathrm{i}} \in G F\left(q_{i}\right)\right\}
$$

The set $A \subseteq\{0,1\}^{\mathrm{n}}$ represents the general mean, main factors, and interactive factors included in the model.

(For example, consider $A \subseteq\{000,100,010,001,110\}$.Then, $000,100,010,001,110$ indicate the general mean, main factor of $F_{1}$, main factor of $F_{2}$, main factor of $F_{3}$, and interactive factor of $F_{1}$ and $F_{2}$, respectively.) The model includes a random error

$\epsilon_{x}$ satisfying the expected value $E\left(\epsilon_{x}\right)=0$ and constant variance $\sigma^{2}$.

In addition, it is usually assumed that the set $A$ satisfies the following monotonicity condition [2].

Definition 2: Monotonicity

$$
a \in A \rightarrow a^{\prime} \in A \quad \forall a^{\prime}\left(\boldsymbol{a}^{\prime} \sqsubseteq \boldsymbol{a}\right),
$$

where $\boldsymbol{a}=\left(a_{1}, a_{2}, \cdots, a_{n}\right), \boldsymbol{a}^{\prime}=\left(a_{1}^{\prime}, a_{2}^{\prime}, \cdots, a_{n}^{\prime}\right)$ and $\boldsymbol{a}^{\prime} \subseteq \boldsymbol{a}$ means that if $a_{i}=0$ then $a^{\prime}{ }_{i}=0, i=1,2, \cdots, n$.

\section{Example 3:}

Consider $A=\{00000,10000,01000,00100,00010,00001,11000,10100,10010\}$.

Since the set $A$ satisfies (11), $A$ is monotonic. 
Next, let $\boldsymbol{w}=\left(w_{1}, w_{2}, \cdots, w_{n}\right)$. The main effect of $F_{i}$ is represented by $\left\{f_{\boldsymbol{w}} \mid w_{i} \neq 0\right.$ and $w_{k}=$ 0 for $k \neq i\}$. The interaction of $F_{i}$ and $F_{j}$ is represented by $\left\{f_{w} \mid w_{i} \neq 0\right.$ and $w_{j} \neq 0$ and $w_{k}=$ 0 for $k \neq i, j\}$

\section{Example 4:}

Consider $A$ given in Example 3 and $q_{1}=2, q_{i}=3, i=2, \ldots, 5$. Then, $I_{A}$ is given by

$$
\begin{gathered}
I_{A}=\{00000,10000,01000,02000,00100,00200,00010,00020, \\
00001,00002,11000,12000\}
\end{gathered}
$$

For example, the main effect of $F_{1}$ is represented by $f_{10000}$, and the interaction of $F_{1}$ and $F_{2}$ is represented by $f_{11000}$ and $f_{12000}$.

In experimental design, we are given a model of the experiment. In other words, we are given a set $A \subseteq\{0,1\}^{n}$. Then, we determine a set of level combinations $x \in X, X \subseteq \prod_{i=1}^{n} G F\left(q_{i}\right)$. The set $X$ is called a design. Next, we perform a set of experiments according to the design $X$ and estimate the effects from the result, $\{(\boldsymbol{x}, y(\boldsymbol{x})) \mid \boldsymbol{x} \in X\}$.

An important standard for evaluating designs is the maximum of the variances of the unbiased estimators of effects calculated from the result of the experiments. It is known that, for a given number of experiments, this criterion is minimized in an orthogonal design [6].

\subsection{Orthogonal Designs}

In this subsection, a definition of Orthogonal Designs for the generalized model is provided.

\section{Definition 3: Orthogonal Designs}

At first, define $v(\boldsymbol{a})=\left\{i \mid a_{i} \neq 0,1 \leq i \leq n\right\}$.

For $\boldsymbol{a}_{\mathbf{1}}=\left(a_{11}, a_{12}, \ldots, a_{1 n}\right), \boldsymbol{a}_{\mathbf{2}}=\left(a_{21}, a_{22}, \ldots, a_{2 n}\right) \in\{0,1\}^{n}$, the addition of vectors $\boldsymbol{a}_{\mathbf{1}}$ and $\boldsymbol{a}_{\mathbf{2}}$ is defined by $\boldsymbol{a}_{\mathbf{1}}+\boldsymbol{a}_{\mathbf{2}}=\left(a_{11} \oplus a_{21}, a_{12} \oplus a_{22}, \ldots, a_{1 n} \oplus a_{2 n}\right)$, where $\oplus$ is the exclusive or operation.

An orthogonal design $C^{\perp}$ for $A \subseteq\{0,1\}^{n}$ is satisfies the condition that for any $\boldsymbol{a}, \boldsymbol{a}^{\prime} \in A$,

$$
\begin{aligned}
& \left|C_{i_{1}, \ldots, i_{m}}^{\perp}\left(\varphi_{1}, \ldots, \varphi_{m}\right)\right|=\frac{\left|C^{\perp}\right|}{q_{i_{1}} q_{i_{2}} \ldots q_{i_{m}}}, \\
& \varphi_{1} \in G F\left(q_{i_{1}}\right), \ldots, \varphi_{m} \in G F\left(q_{i_{m}}\right)
\end{aligned}
$$

where $i_{1}, \ldots, i_{m}$ are defined by $v\left(\boldsymbol{a}+\boldsymbol{a}^{\prime}\right)=\left\{i_{1}, \ldots, i_{m}\right\}$, and $C_{i_{1}, \ldots, i_{m}}^{\perp}\left(\varphi_{1}, \ldots, \varphi_{m}\right)=\left\{\boldsymbol{x} \mid x_{i_{1}}=\right.$ $\left.\varphi_{1}, \ldots, x_{i_{m}}=\varphi_{m}, x \in C^{\perp}\right\}$.

\section{Example 5:}

Consider $A$ given in Example 3 and $q_{1}=2, q_{i}=3, i=2, \ldots, 5$.

Then, an orthogonal design $C^{\perp}$ for $A$ is given as follows. 
Table 1. Example of orthogonal design $C^{\perp}$.

\begin{tabular}{|c|c|c|c|c|c|}
\hline & \multirow{2}{*}{\multicolumn{5}{|c|}{$\begin{array}{ccccc}x_{1} & x_{2} & x_{3} & x_{4} & x_{5} \\
0 & & 0 & 0 & 0\end{array}$}} \\
\hline 1 & & & & & \\
\hline 2 & 0 & 0 & 1 & 1 & 1 \\
\hline 3 & 0 & 0 & 2 & 2 & 2 \\
\hline 4 & 0 & 1 & 0 & 0 & 1 \\
\hline 5 & 0 & 1 & 1 & 1 & 2 \\
\hline 6 & 0 & 1 & 2 & 2 & 0 \\
\hline 7 & 0 & 2 & 0 & 1 & 0 \\
\hline 8 & 0 & 2 & 1 & 2 & 1 \\
\hline 9 & 0 & 2 & 2 & 0 & 2 \\
\hline 10 & 1 & 0 & 0 & 2 & 2 \\
\hline 11 & 1 & 0 & 1 & 0 & 0 \\
\hline 12 & 1 & 0 & 2 & 1 & 1 \\
\hline 13 & 1 & 1 & 0 & 1 & 2 \\
\hline 14 & 1 & 1 & 1 & 2 & 0 \\
\hline 15 & 1 & 1 & 2 & 0 & 1 \\
\hline 16 & 1 & 2 & 0 & 2 & 1 \\
\hline 17 & 1 & 2 & 1 & 0 & 2 \\
\hline 18 & 1 & 2 & 2 & 1 & 0 \\
\hline
\end{tabular}

The Hamming weight $H w(\boldsymbol{a})$ of a vector $\boldsymbol{a}=\left(a_{1}, a_{2}, \cdots, a_{n}\right)$ is defined as the number of nonzero components. As a special case, if $A=\left\{\boldsymbol{a} \mid H w(\boldsymbol{a}) \leq t, \boldsymbol{a} \in\{0,1\}^{n}\right\} \cdot C^{\perp}$ corresponds to the set of rows of a subarray in a mixed level orthogonal array of strength $2 t$ [1]. Hence, $C^{\perp}$ can be easily obtained by using the results of orthogonal arrays.

However, because it is generally not easy to construct an orthogonal design for $A$, it is important to consider efficiency in making the algorithm to produce the design. However, because the main purpose of this paper is not to construct the orthogonal design, the algorithm is not included in this paper.

\section{SAMPLING THEOREM FOR FUNCTIONS OVER GALOIS FIELD DOMAINS FOR EXPERIMENTAL DESIGN}

In this section, I provide a sampling theorem for bandlimited functions over Galois field domains, which is applicable to the experimental design model in which each factor has a different number of levels.

\subsection{Bandlimited Functions}

The range of frequencies of $f$ is defined by a bounded set $I \subset \prod_{i=1}^{n} G F\left(q_{i}\right)$. Then, $f_{w}=0$ for all $\boldsymbol{w} \in \prod_{i=1}^{n} G F\left(q_{i}\right) \backslash I$. Any function whose range of frequencies is confined to a bounded set $I$ is referred to as bandlimited to $I$. 


\subsection{A Sampling Theorem for Bandlimited Functions over $\prod_{i=1}^{n} G F\left(q_{i}\right)$ Domains}

Theorem 1:

Suppose a set $A$ is monotonic and $f(\boldsymbol{x})$ is expressed as

$$
f(x)=\sum_{w \in I_{A}} f_{w} \chi_{w}(x),
$$

where $I_{A}=\left\{\left(\mathrm{b}_{1} \mathrm{a}_{1}, \mathrm{~b}_{2} \mathrm{a}_{2}, \ldots, \mathrm{b}_{\mathrm{n}} \mathrm{a}_{\mathrm{n}}\right) \mid \boldsymbol{a} \in A, \mathrm{~b}_{\mathrm{i}} \in G F\left(q_{i}\right)\right\}$. Then, the Fourier coefficients can be computed by

$$
f_{\boldsymbol{w}}=\frac{1}{\left|C^{\perp}\right|} \sum_{\boldsymbol{x} \in C^{\perp}} f(\boldsymbol{x}) \chi_{\boldsymbol{w}}^{*}(\boldsymbol{x}),
$$

where $C^{\perp}$ is an orthogonal design for $A$.

The proof of Theorem 1 requires the following three lemmas.

Lemma 1:

For any non principal character $\chi$ of $H$,

$$
\sum_{\boldsymbol{h} \in H} \chi(\boldsymbol{h})=0,
$$

Proof: This follows immediately [5, Lemma 2.4].

\section{Lemma 2:}

Suppose a set $A$ is monotonic, and $C^{\perp}$ is an orthogonal design for $A$.

Then, for $\boldsymbol{w}, \boldsymbol{z} \in I_{A}$,

$$
\begin{gathered}
\left|C_{i_{1}, \ldots, i_{m}}^{\perp}\left(\varphi_{1}, \ldots, \varphi_{m}\right)\right|=\frac{\left|C^{\perp}\right|}{q_{i_{1}} q_{i_{2}} \ldots q_{i_{m}}}, \\
\varphi_{1} \in G F\left(q_{i_{1}}\right), \ldots, \varphi_{m} \in G F\left(q_{i_{m}}\right)
\end{gathered}
$$

where $i_{1}, \ldots, i_{m}$ are defined by $v(\mathbf{z}-w)=\left\{i_{1}, \ldots, i_{m}\right\}$.

Proof: Let $S_{A}=\left\{v\left(\boldsymbol{a}+\boldsymbol{a}^{\prime}\right) \mid \boldsymbol{a}, \boldsymbol{a}^{\prime} \in A\right\}$. Because a set $A$ is monotonic, $v(\boldsymbol{z}-w) \in S_{A}$ holds for $\boldsymbol{w}, \boldsymbol{z} \in I_{A}$. Hence, by the definition of $C^{\perp}$, equation (16) holds.

Lemma 3:

Suppose a set $A$ is monotonic, and $C^{\perp}$ is an orthogonal design for $A$. Then, 


$$
\sum_{\boldsymbol{x} \in C^{\perp}} \chi_{\boldsymbol{z}}(\boldsymbol{x}) \chi_{w}^{*}(\boldsymbol{x})=\left\{\begin{array}{lr}
\left|C^{\perp}\right|, & \boldsymbol{w}=\mathbf{z} \\
0, & \text { otherwise }
\end{array}\right.
$$

for all $\boldsymbol{z} \in I_{A}$.

Proof: If $\boldsymbol{w}=\mathbf{z}$, then $\chi_{\boldsymbol{z}}(\boldsymbol{x}) \chi_{\boldsymbol{w}}^{*}(\boldsymbol{x})=\chi_{\mathbf{0}}(\boldsymbol{x})=1$ for any $\boldsymbol{x}$. Hence, $\sum_{\boldsymbol{x} \in C^{\perp}} \chi_{\boldsymbol{z}}(\boldsymbol{x}) \chi_{\boldsymbol{w}}^{*}(\boldsymbol{x})=\left|C^{\perp}\right|$.

Next, consider the case that $\boldsymbol{w} \neq \boldsymbol{z}$. Define $\boldsymbol{u}=\boldsymbol{z}-\boldsymbol{w}$ and let $v(\boldsymbol{u})=\left\{i_{1}, \ldots, i_{m}\right\}$. Then,

$$
\begin{aligned}
\sum_{\boldsymbol{x} \in C^{\perp}} \chi_{\boldsymbol{z}}(\boldsymbol{x}) \chi_{\boldsymbol{w}}^{*}(\boldsymbol{x}) & =\sum_{\boldsymbol{x} \in C^{\perp}} \chi_{\boldsymbol{u}}(\boldsymbol{x}) \\
& =\sum_{\boldsymbol{x} \in C^{\perp}} \chi_{u_{i_{1}, \ldots, u_{i_{m}}}}\left(x_{i_{1}}, \ldots, x_{i_{m}}\right) \\
& =\frac{\left|C^{\perp}\right|}{q_{i_{1}} q_{i_{2}} \ldots q_{i_{m}}}\left(\sum_{\boldsymbol{h} \in \prod_{j=1}^{m} G F\left(q_{i_{j}}\right)} \chi_{u_{i_{1}, \ldots, u_{i_{m}}}}(\boldsymbol{h})\right)
\end{aligned}
$$

where $\chi_{u_{i j}}\left(x_{i_{j}}\right)=1$ for $u_{i_{j}}=0$, was used for the transformation from (18) to (19), and Lemma 2 was used for the transformation from (19) to (20). Then, by (20) and Lemma 1, $\sum_{x \in C^{\perp}} \chi_{z}(\boldsymbol{x}) \chi_{w}^{*}(\boldsymbol{x})=0$ is obtained.

Proof of Theorem 1: The right hand side of Equation (14) is given by

$$
\begin{aligned}
\frac{1}{\left|C^{\perp}\right|} \sum_{\boldsymbol{x} \in C^{\perp}} f(\boldsymbol{x}) \chi_{\boldsymbol{w}}^{*}(\boldsymbol{x}) & =\frac{1}{\left|C^{\perp}\right|} \sum_{\boldsymbol{x} \in C^{\perp}}\left(\sum_{\boldsymbol{z} \in I_{A}} f_{\boldsymbol{z}} \chi_{\mathbf{z}}(\boldsymbol{x})\right) \chi_{\boldsymbol{w}}^{*}(\boldsymbol{x}) \\
& =\frac{1}{\left|C^{\perp}\right|} \sum_{\boldsymbol{z} \in I_{A}} f_{\boldsymbol{z}}\left(\sum_{\boldsymbol{x} \in C^{\perp}} \chi_{\boldsymbol{z}}(\boldsymbol{x}) \chi_{\boldsymbol{w}}^{*}(\boldsymbol{x})\right) \\
& =f_{\boldsymbol{w}}
\end{aligned}
$$

where Lemma 3 was used for the transformation from (21) to (22). Hence, Theorem 1 is obtained.

Theorem 1 is applicable to the generalized model given in Definition 1. When we experiment according to an orthogonal design $C^{\perp}$, we can obtain unbiased estimators of the $f_{w}$ in (9) using Theorem 1 and the assumption that $\epsilon_{\boldsymbol{x}}$ is a random error with zero mean,

$$
\hat{f}_{\boldsymbol{w}}=\frac{1}{\left|C^{\perp}\right|} \sum_{\boldsymbol{x} \in C^{\perp}} f(\boldsymbol{x}) \chi_{\boldsymbol{w}}^{*}(\boldsymbol{x})
$$


Hence, the parameters can be estimated by using Fourier transforms.

\section{RELATIONSHIP BETWEEN THE SAMPLING THEOREM AND ORTHOGONAL ARRAYS}

Experiments are frequently conducted according to an orthogonal array. Here, the relationship between the proposed sampling theorem and orthogonal arrays will be provided.

At first, mixed level orthogonal arrays of strength $t$ are defined as follows.

\section{Definition 4: Orthogonal Arrays of strength t [1]}

An Orthogonal Array of strength $t$ is $N \times n$ matrix whose $i$-th column contains $q_{i}$ different factor-levels in such a way that, for any $\$ \mathrm{t} \$$ columns, every $\$ \mathrm{t} \$$-tuple of levels appears equally often in the matrix.

The $N$ rows specify the different experiments to be performed.

Next, the definition of orthogonal arrays of strength $t$ can be generalized by using a bounded set $A$ instead of the strength $t$. The definition of the generalized mixed level orthogonal arrays is provided as follows.

\section{Definition 5: Orthogonal Arrays for A}

An orthogonal array for $A$ is an $N \times n$ matrix whose $i$-th column contains $q_{i}$ different factorlevels in such a way that, for any $\boldsymbol{a}, \boldsymbol{a}^{\prime} \in A$, and for any $m$ columns which are $i_{1}$-th column, ..., $i_{m}$-th column, where $i_{1}, \ldots, i_{m}$ are defined by $v\left(\boldsymbol{a}+\boldsymbol{a}^{\prime}\right)=\left\{i_{1}, \ldots, i_{m}\right\}$, every $m$-tuple of levels appears equally often in the matrix.

If $A=\left\{\boldsymbol{a} \mid H w(\boldsymbol{a}) \leq t, \boldsymbol{a} \in\{0,1\}^{n}\right\}$, an orthogonal array for $A$ is identical to a mixed level orthogonal array of strength $2 t$. In other words, Definition 4 is a special case of Definition 5.

Moreover, by Definition 3 and Definition 5, it is clear that the set of rows of an orthogonal array for $A$ is an orthogonal design $C^{\perp}$ for $A$. Hence the following Corollary is obtained from Theorem 1 immediately.

\section{Corollary 1:}

Suppose a set $A$ is monotonic and $f(\boldsymbol{x})$ is expressed as

$$
f(x)=\sum_{w \in I_{A}} f_{w} \chi_{w}(x),
$$

where $I_{A}=\left\{\left(\mathrm{b}_{1} \mathrm{a}_{1}, \mathrm{~b}_{2} \mathrm{a}_{2}, \ldots, \mathrm{b}_{\mathrm{n}} \mathrm{a}_{\mathrm{n}}\right) \mid \boldsymbol{a} \in A, \mathrm{~b}_{\mathrm{i}} \in G F\left(q_{i}\right)\right\}$. Then, the Fourier coefficients can be computed by

$$
f_{\boldsymbol{w}}=\frac{1}{\left|C^{\perp}\right|} \sum_{\boldsymbol{x} \in C^{\perp}} f(\boldsymbol{x}) \chi_{w}^{*}(\boldsymbol{x}),
$$


where $C^{\perp}$ is the set of rows of an orthogonal array for $A$ defined in Definition 5 and $\left|C^{\perp}\right|=N$.

This corollary shows the relationship between the proposed sampling theorem and orthogonal arrays.

\section{CONCLUSiOnS}

In this paper, I have generalized the sampling theorem for bandlimited functions over $G F(q)^{n}$ domains to one over $\prod_{i=1}^{n} G F\left(q_{i}\right)$ domains. The generalized theorem is applicable to the experimental design model in which each factor has a different number of levels and enables us to estimate the parameters in the model by using Fourier transforms. I have also provided the relationship between the proposed sampling theorem and orthogonal arrays.

\section{ACKNOWLEDGEMENTS}

This paper was partially supported by the Academic Committee of Yokohama College of Commerce.

\section{REFERENCES}

[1] A.S. Hedayat, N.J.A. Sloane and J. Stufken, (1999) Orthogonal Arrays: Theory and Applications, Springer.

[2] T. Okuno and T. Haga, (1969) Experimental Designs, Baifukan, Tokyo.

[3] A.V. Oppenheim and R.W. Schafer, (1975) Digital Signal Processing, Prentice-Hall.

[4] R.S. Stankovic and J. Astola, (2007) "Reading the Sampling Theorem in Multiple-Valued Logic: A journey from the (Shannon) sampling theorem to the Shannon decomposition rule," in Proc. 37th Int. Symp. on Multiple-Valued Logic, Oslo, Norway.

[5] E.M. Stein and R. Shakarchi, (2003) Fourier Analysis: An Introduction, Princeton University Press.

[6] I. Takahashi, (1979) Combinatorial Theory and its Application, Iwanami Syoten, Tokyo.

[7] H. Toutenburg and Shalabh, (2009) Statistical Analysis of Designed Experiments (Third Edition), Springer.

[8] Y. Ukita, T. Saito, T. Matsushima and S. Hirasawa, (2010) "A Note on a Sampling Theorem for Functions over GF(q)^n Domain,” IEICE Trans. Fundamentals, Vol.E93-A, no.6, pp.1024-1031.

[9] Y. Ukita and T. Matsushima, (2011) "A Note on Relation between the Fourier Coefficients and the Effects in the Experimental Design," in Proc. 8th Int. Conf. on Inf., Comm. and Signal Processing, pp.1-5.

[10] Y. Ukita, T. Matsushima and S. Hirasawa, (2012) "A Note on Relation Between the Fourier Coefficients and the Interaction Effects in the Experimental Design," in Proc. 4th Int. Conf. on Intelligent and Advanced Systems, Kuala Lumpur, Malaysia, pp.604-609. 
[11] Sharma, S., Tim, U. S., Wong, J., Gadia, S., and Sharma, S. (2014) "A Brief Review on Leading Big Data Models," Data Science Journal, 13(0), pp.138-157.

[12] Sharma, S., Shandilya, R., Patnaik, S., and Mahapatra, A. (2015) Leading NoSQL models for handling Big Data: a brief review, International Journal of Business Information Systems, Inderscience.

\section{AUTHORS}

Yoshifumi Ukita has been a professor of the Department of Management Information at Yokohama College of Commerce, Kanagawa, Japan since 2011. His research interests are artificial intelligence, signal processing and experimental designs. He is a member of the Information Processing Society of Japan, the Japan Society for Artificial Intelligence and IEEE. 